\title{
Inducing the familiarity effect
}

\author{
ROBERT M. SCHINDLER, ARNOLD D. WELL, and ALEXANDER POLLATSEK \\ University of Massachusetts, Amherst, Massachusetts 01002
}

\begin{abstract}
The task required subjects to judge whether members of six-letter consonant string pairs were physically identical. Some (repeated) strings were presented on each of the 16 training sessions, while other (nonrepeated) strings were presented only during a single session. An advantage in matching time of repeated strings over nonrepeated strings was obtained which increased in a roughly linear fashion during training and which persisted for at least 7 weeks after the last training session. Detailed analyses of the different responses and the results of several transfer tasks suggest that perceptual processing is indeed facilitated by acquired information about which letters usually follow others and about the positions in which these sequences of letters are likely to occur, but provide no evidence that this facilitation derives from the formation of visual features which are larger than single letters.
\end{abstract}

Recently, there has been much research demonstrating that familiar letter strings (e.g., words, acronyms) and letter strings with at least some familiar orthographic patterns (e.g., pronounceable nonwords) can be perceived more rapidly or more accurately than strings of unrelated letters (see review articles by Baron, in press; Krueger, 1975; Smith \& Spoehr, 1974). It is usually presumed that this perceptual superiority of certain letter strings, the familiarity effect, is due to the acquisition of information about letter strings during our past experience with them. Yet, few of the many studies of the familiarity effect have experimentally manipulated the subject's experience with letter strings.

Baron (1974, Experiment III) manipulated experience with letter strings and demonstrated the effect of this experience on letter-string perception using a forced-choice tachistoscopic recognition task. He constructed two sets of two-letter consonant strings, the members of each set differing from the members of the other set only in letter order. Each subject received a pretest on both sets of letter strings, at least three sessions of practice on only one of the two sets, and then a posttest on both sets of letter strings. Baron found that while accuracy improved on both letter-string sets, the improvement was larger on the set of letter strings that had been presented during the three practice sessions.

The present study was an attempt to experimentally induce perceptual superiority in a set of letter strings whose length was greater than digrams and compar-

This research was supported in part by National Science Foundation Social Science Development .Grant GU-4041, and in part by a grant from the Research Council of the University of Massachusetts to the second author. The authors would like to thank Jonathan Baron, Lester Krueger, and Edward Smith for their helpful comments on this research. Requests for reprints should be sent to Robert M. Schindler, Department of Psychology, University of Massachusetts, Amherst, Massachusetts 01002 . able to that of most words. Further, we hoped to be able to provide subjects sufficient experience with a set of letter strings to cause a familiarity effect comparable in magnitude and permanence to the familiarity effects shown by words, acronyms, and pronounceable nonwords (e.g., Baron \& Thurston, 1973; Eichelman, 1970; Henderson, 1974). If, by our technique, such a familiarity effect could be induced in arbitrarily chosen letter strings, we would then have a useful paradigm for studying the path of acquisition of perceptually useful letter-string information. Such a procedure would provide the opportunity to use transfer tasks to investigate the question of what is learned during this acquisition. Also, since letter strings could be used which are not normally encountered in everyday experience, this technique could tell us how perceptual learning is retained over time.

The present study consisted of 23 experimental sessions, which were divided into three parts: (a) 16 training sessions, (b) 4 transfer sessions, and (c) 3 retention sessions. During each session, the subject's task was to press a key to respond same or different to pairs of six-letter strings which were presented one above the other. The subject's reaction time (RT) for each pair was recorded and constituted our measure of the speed of perception. Using this matching task, Eichelman (1970), Schindler, Well, and Pollatsek (1974), and Well, Pollatsek, and Schindler (1975) found familiarity effects for pairs of six-letter strings which ranged from 200 to $550 \mathrm{msec}$.

To insure that all of the letter strings used in the study would be highly unfamiliar, all were constructed from a set of 12 consonants. Out of 46,080 possible combinations, 6 were chosen randomly (individually for each subject) as the ones to be repeated during training. During each training session, a repeated ${ }^{\perp}$ letter string was shown on half the trials and a nonrepeated string was shown on the 
other half of the trials. The nonrepeated strings were selected randomly before each trial block from the thousands of possibilities. In this way, we were able to obtain a continuous measure of the acquisition of the familiarity effect over the course of 16 training sessions.

The first transfer session was the same as a training session except that when the pairs of letter strings differed, they differed in novel ways. This session was designed to test certain versions of the hypothesis that the familiarity effect in the matching task is due to facilitation of the matching process only. Such models of the familiarity effect predict a decrease in the familiarity effect if the letters involved in, and hence the features relevant to a different judgment are not the same as those present during acquisition of the familiarity effect. The second transfer session differed from a training session in that the letterstring pairs were presented as two vertical columns rather than as two horizontal rows. If the acquisition of information about visual features which are larger than single letters plays a role in the familiarity effect, then altering such between-letter visual features by presenting the strings vertically should result in a decreased familiarity effect.

In the third transfer session, subjects were presented with "partially familiar" letter strings in which either the first three letters or the last three letters were the same as those of a repeated string. Although it is possible that the familiarity effect results from the learning of only the probabilities that one letter follows another letter, Gibson (1969) and others have suggested that stored information about the position in the string of a familiar letter sequence also plays a role in the familiarity effect. To test this notion, the third transfer session included some partially familiar strings where the familiar three-letter sequences were moved three letter positions to the left or right to produce strings which had a familiar three-letter sequence in an unfamiliar position in the string. If the matching RT to those partially familiar strings which preserve the position of the familiar letter sequence is smaller than that for otherwise similar strings in which the position of the familiar sequence is altered, then stored position information must play a role in the perceptual facilitation of familiar letter strings. (For the fourth transfer session, see Note 2.)

The first retention session, which was 1 day after the last transfer session, was designed to provide a baseline for the later retention sessions in case the induced familiarity effect had decayed during the 3 days of transfer sessions. The second retention session was run 1 week after the baseline session, and the third was run 7 weeks after the baseline session. These were run to test the permanence of the perceptual changes acquired during the training sessions.

\section{METHOD}

\section{Subjects}

Four University of Massachusetts purchology students served as paid subjects. All tour were right-handed.

\section{Apparatus and Manner of Stimulus Presentation}

A Hewlett-Packard $2114 \mathrm{~B}$ computer controlled the presentation of capital letters un an HP-1300A X-Y display oscillosicope. It also recorded the subject's responses and measured the response time in milliseconds. The subjects were run individually and sat approximately $1.5 \mathrm{~m}$ from the oscilloscope steen, in a dimly lit room.

The display for a single trial consisted of two siv-letter strings with one string directly above the other. Each of the letters in a string was constructed by illuminating the appropriate pattern of points in a matrix 13 points high and 9 points wide. A single letter was $1.00 \mathrm{~cm}$ high and $.87 \mathrm{~cm}$ wide, and there was $.21 \mathrm{~cm}$ between letters. The letter strings were $6.28 \mathrm{~cm}$ long and subtended $2 \mathrm{deg}$ $22 \mathrm{~min}$ of visual angle. The vertical distance between the two strings was $1.74 \mathrm{~cm}$. and hence the total tertical visual angle subtended was 1 deg $30 \mathrm{~min}$.

\section{Procedure}

The experiment consisted of 2345 -min sessions. The procedure was essentially the same for all sessions except Session $21 .^{2}$ The subjects were instructed to respond as quiskly and accurately as possible and were told in general terms what kinds of stimuli they would be seeing. Each session began with at least one practice block consisting of 24 pairs of random six-digit strings. Following the practice, each subject was given four blocks, each consisting of 48 pairs of six-letter strings. The subject initiated each block when he was ready; the interval between trial blocks varied from $1102 \mathrm{~min}$.

A single trial began with the presentation of a fixation point $\left(a{ }^{\prime}+"\right)$ in the center of the screen for $1 \mathrm{sec}$ and was followed by a blank screen for 500 msec. Then the pair of strings was displayed. The strings remained on until the subject responded by pressing the appropriate key on the response panel in front of him. Different responses were always made with the right hand. Following the subject's response. feedback, which consisted of the RT in milliseconds for a correct response and the word "error" for an incorrect response, was displayed in the lower left-hand corner of the screen. When the subject responded incorrectly, the sequence of the trial block paused until the subject indicated. by pressing a middle key, that he was ready to continue. The interval between the feedback for a correct response and the beginning of the next trial was 1 sec.

\section{Stimuli and Design}

The consonants comprising the letter strings used in this study were chosen from the following set of six conjugute puirs: BG, CJ, DP, FH, KM, and NL. Fach letter string was a sequence of six consonants, one chosen from each conjugate pair. When a pair of letter strings was presented during a trial, each letter was paired either with itself or with its conjugate. This was true for both repeated and nonrepeated strings. All of the string pairs in the study which were different differed in only one letter position. and the location of the difference was randomly varied over the six letter positions.

For each of the four subjects, a separate set of six repeated strings was chosen. This selection was random, except for the constraint above that one member of each conjugate pair was represented in every string) and the constraints that (1) no letter could appear more than once in a single position in the set of six repeated strings, and (2) no sequence of siv letters could appear more than once in the set of six repeated strings. The four sets of six repeated strings were chosen before the experiment began and remained fixed throughoul the 23 sessions. The sets of repeated strings used for each subject are presented in Table 1. 
Table 1

The Sets of Repeated Strings Used for Each Subject

\begin{tabular}{cccc}
\hline Subject 1 & Subject 2 & Subject 3 & Subject 4 \\
\hline NJPFMG & GPCFLK & MNFBDC & JHLMDB \\
CFGLDK & DFKBJN & LBCKHP & FMBCNP \\
PBJMNF & CMPLGF & FJNDMB & LGHDJM \\
HNKJGD & HGNPKJ & GMPNCH & KJPHBN \\
KDNBFJ & NJGMFP & PHMJBN & PNJGMH \\
BMFPJL & MLFCPB & JDBFNK & GDMLHC \\
\multicolumn{4}{c}{ Examples of Nonrepeated String Sets } \\
\hline MDBFNC & \multicolumn{2}{c}{ BDMCNF } \\
GFLKDC & \multicolumn{2}{|l}{ JFGNKD } \\
CNFGPK & \multicolumn{2}{c}{ FMCBPL } \\
BKDFJN & NPGJMF \\
CPKLFB & HLJKPG \\
LCFPMB & DMNGFJ
\end{tabular}

A "repeated" trial block consisted of random selections (by computer) from the set of six repeated letter strings. On the average, half of the letter-string pairs were the same and half were different.

A "nonrepeated" trial block consisted of random selections from a set of six nonrepeated letter strings which were chosen anew before each nonrepeated block. Each set of six nonrepeated strings was formed by random selection from the 46,080 possible strings, except for the previously mentioned constraint that a member of eact conjugate pair be represented in every string and the further constraint that the string could not be identical to any of the six repeated strings chosen for that particular subject. Two examples of a set of nonrepeated strings are presented in Table 1.

A "mixed" trial block consisted of random selections from both the set of six repeated strings and a newly chosen set of six nonrepeated strings. Otherwise, a mixed block was constructed in the same ways as the repeated and nonrepeated blocks.

Sessions 1 through 16 were training sessions. The experimental trials in Session 1 consisted of one repeated block, one nonrepeated block, and two mixed blocks; in Sessions 2-16, the experimental trials consisted of two blocks of repeated strings (R) and two blocks of nonrepeated strings $(N)$. The order of the blocks for a session alternated between RNRN and NRNR. At the end of Session 16, each subject was given a list of 24 six-letter strings typed in uppercase letters. The subject was told that he had seen
6 of the strings on the list many times during the experiment (since they were his six repeated strings), while the other 18 strings were probably completely novel. ${ }^{3}$ He was asked to circle the 6 strings he had seen before, and was given unlimited time to make his choices.

Sessions 17 through 19 were transfer sessions. Session 17 was identical to Sessions 2-16, except that new conjugate pairings were assigned to each subject (for example, one new set of conjugate pairs was BC, GP, JD, FK, NM, and $H L$ ). On Session 18, the letter strings were presented in vertical columns. Session 19 consisted of four mixed blocks of the four types of partially familiar strings: those in which the first three letters were familiar and

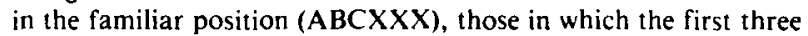
letters were familiar but in an unfamiliar position (DEFXXX), those in which the last three letters were familiar and in the familiar position (XXXDEF), and those in which the last three letters were familiar but in an unfamiliar position (XXXABC).

Session 20 was the baseline for the retention sessions and consisted of two repeated and two nonrepeated blocks. After Session 20 , subjects were asked to give written answers to some open-ended questions concerning their matching strategies and their general reactions to the experimental situation. Sessions 22 and 23 were the 1-week and 7-week retention sessions; these, like Session 1, consisted of one repeated block, one nonrepeated block, and two mixed blocks.

The first 21 sessions were on consecutive weekdays beginning with a Monday. Session 22 was on the Friday following Session 21, and Session 23 was on the sixth Friday after Session 22.

\section{RESULTS AND DISCUSSION}

\section{Training Sessions}

In all of the following analyses, latencies from only the correct responses were used. Mean RTs were calculated for each subject at each level of familiarity (repeated, nonrepeated), response (same, different), and sessions (1-16). An analysis of variance on these means showed a significant decrease in mean RT over the course of the training sessions, $F(15,45)=16.67$, $p<.001$. This decrease is illustrated in Figure 1 .

Repeated letter strings were matched more rapidly than nonrepeated strings, $F(1,3)=66.08, p<.005$, and the size of this difference varied over sessions,
Figure 1. Mean RT and error rate for each experimental session.

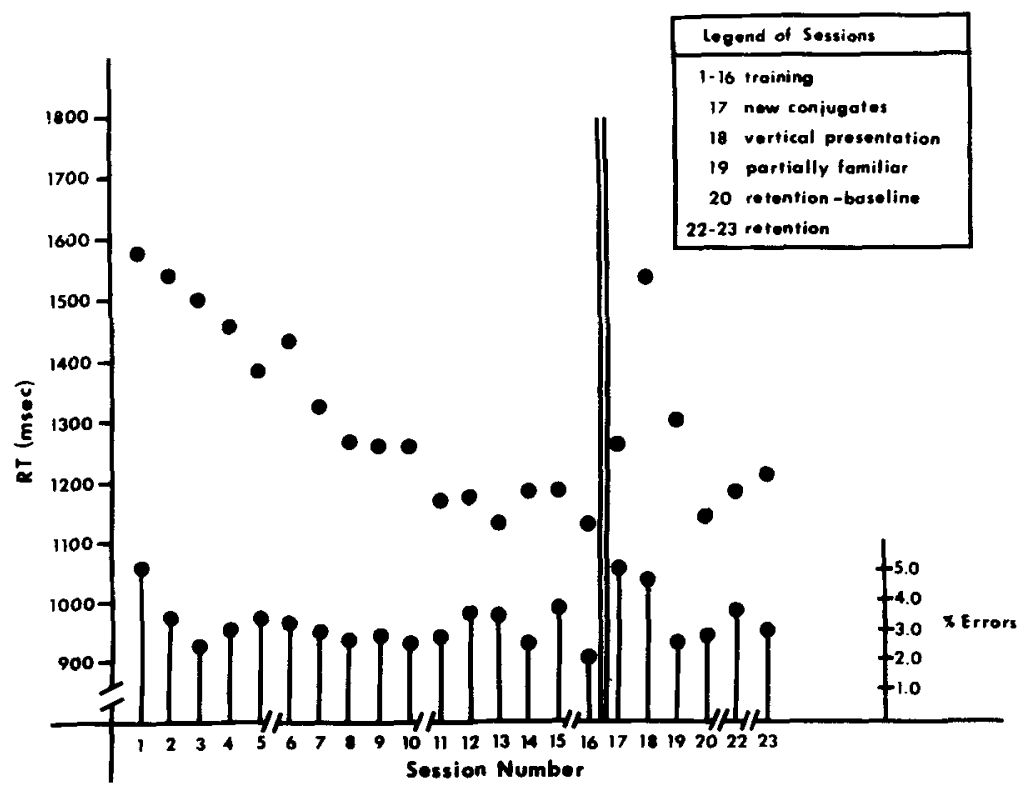


$F(15,45)=1.97, p<.05$. To further examine this effect of letter-string familiarity, a linear trend analysis was performed on the data. The linear component of the Familiarity by Sessions interaction was found to be significant, $F(1,3)=17.06, \mathrm{p}<.05$, and the remainder of the interaction was not significant, $F(14,42)=1.27, p<.20$. Thus, it appears that the $\mathrm{RT}$ advantage of repeated over nonrepeated strings tended to rise in a linear fashion. To illustrate this result, the mean familiarity effect for overlapping groups of three sessions is presented in Figure 2. This smoothed function is quite linear, and it suggests that the perceptual advantage resulting from letter-string repetition increased steadily over the course of the training period. ${ }^{4}$

While the effect of string repetition over the 16 training sessions was smaller for different responses than for same responses, $F(1,3)=40.45, p<.01$, different responses to repeated strings were still significantly faster than those to nonrepeated strings, $F(1,3)=464.96, p<.001$. Further analysis of the different response times showed that position of the difference in the letter string significantly affected both mean RT, $F(5,15)=46.68, p<.001$, and the size of the advantage of repeated over nonrepeated strings, $F(5,15)=5.63, p<.005$. The 50 orm of these relationships can be seen in Figure 3.

The increase of different $R T$ with increasing serial position of the differing letter pair implies that the strings were encoded and/or matched in units smaller than the whole string, and suggests that the units were usually processed serially from left to right, in a self-terminating fashion. The result that the induced familiarity effect for different responses increased with serial position of the difference agrees well with

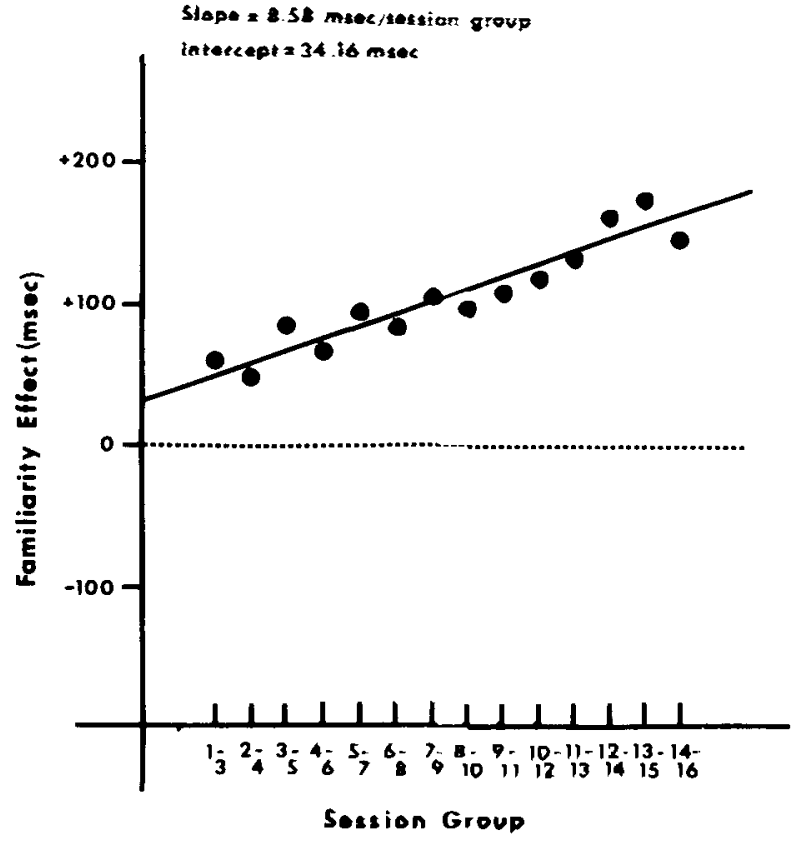

Figure 2. Mean familiarity effect for each group of three training sessions. The best-fitting straight line accounts for $89.1 \%$ of the variance.

a similar finding for word and nonword letter strings (Well, Pollatsek, \& Schindler, 1975). The similarity of these two results supports the argument that the perceptual facilitation induced in the present study is, indeed, a valid laboratory analogue of naturally occurring familiarity effects. Furthermore, the increase of the familiarity effect for same judgments suggests that the familiarity effect is due to the facilitation of a mechanism which can use the

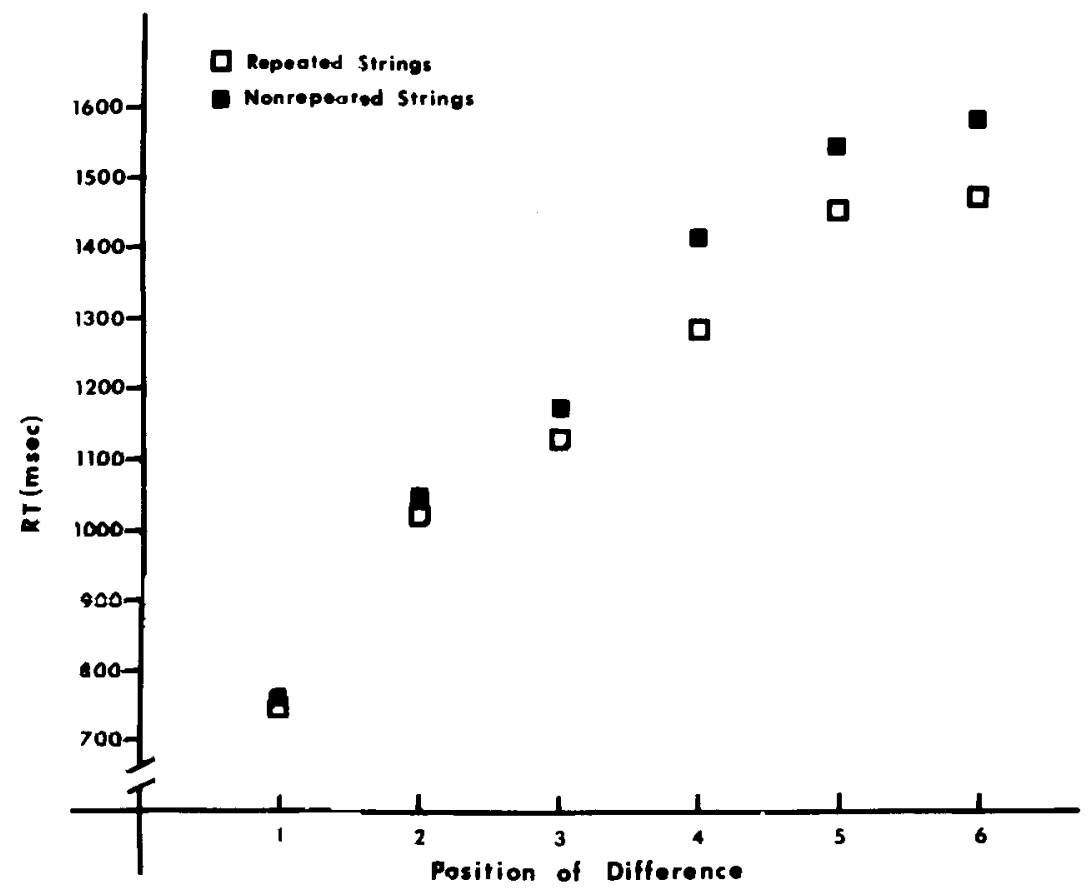

Figure 3. RY for a different response as a function of the position of difference for repealed and nonrepeated stimuli. 
Table 2

Comparison of RT and Familiarity Effect of Transfer and Retention Sessions with Predictions from Training Sessions

\begin{tabular}{|c|c|c|c|c|c|c|c|c|c|}
\hline $\begin{array}{c}\text { Session } \\
\text { Description }\end{array}$ & $\begin{array}{l}\text { Session } \\
\text { Number }\end{array}$ & $\begin{array}{c}\text { Mean } \\
\text { RT }\end{array}$ & $\begin{array}{c}\text { Mean RT } \\
\text { Minus } \\
1156^{*}\end{array}$ & $t(3)$ & $\begin{array}{c}p \\
\text { two-tailed }\end{array}$ & $\begin{array}{c}\text { Mean } \\
F E\end{array}$ & $\begin{array}{c}\text { Mean FE } \\
\text { Minus } \\
161^{* *}\end{array}$ & $t(3)$ & $\underset{\text { two-tailed }}{p}$ \\
\hline New Conjugates & 17 & 1257 & 101 & 1.20 & n.s. & 150 & 11 & .43 & n.s. \\
\hline Vertical Presentation & 18 & 1536 & 379 & 5.99 & .01 & 114 & 47 & 1.35 & n.s. \\
\hline Partjally Familiar & 19 & 1300 & 144 & 1.47 & n.s. & & & & \\
\hline Retention Baseline & 20 & 1141 & -15 & .29 & n.s. & 201 & -40 & 2.84 & .10 \\
\hline 1-Week Retention & 22 & 1177 & 21 & .32 & n.s. & 201 & -40 & .76 & n.s. \\
\hline 7-Week Retention & 23 & 1207 & 51 & .72 & n.s. & 158 & 3 & .06 & n.s. \\
\hline
\end{tabular}

*Mean RT for Sessions $13.16=1156 \mathrm{msec}$.

**Projected familiarity effect for Session $17=161 \mathrm{mset}$.

analysis of initial features to speed the processing of later features (cf. Baron, in press). For example, the extended experience with the repeated strings may have enabled the perceptual processes to use information about which letters are more likely to follow others in a letter string to test specifically for those letters and, therefore, match them more rapidly.

During the training sessions, there were only six letter discriminations possible, one corresponding to each of the conjugate pairs. Since every conjugate pair occurred approximately equally often in each position over the 16 sessions for both repeated and nonrepeated strings, the amount of practice on individual letter-pair discrimination was the same for both. However, if the continual presence of a specific letter context changes the discriminability of a pair (e.g., by allowing the formation of features which are larger than single letters), then one might expect the relative discriminability of the six pairs to be different for the repeated and nonrepeated stimuli. Comparison of the mean RTs of each conjugate pair for repeated and nonrepeated strings showed that there was no significant interaction of letter-string familiarity with discriminability of conjugate pairs, $F<1$. Moreover, the mean RTs of each conjugate pair covaried for repeated and nonrepeated strings, $r=.84, p<.05$. These two results suggest that the existence of an unchanging letter context does not affect the relative discriminability of a letter pair, and thus fail to provide support for the idea that the perceptual superiority of familiar letter strings is due to their being processed in terms of larger visual units.

\section{Transfer and Retention Sessions}

For each subject, the mean RT for Sessions 13-16 was computed, and these means were compared with each subject's mean RT for each of the transfer and retention sessions. The mean familiarity effect for each subject on each of the 16 sessions was computed, and for each subject the best-fitting line through these points was used to derive a projected estimate of the Session 17 familiarity effect. These projections were compared with each subject's mean familiarity effect for each of the transfer and retention sessions (see Table 2). ${ }^{5}$

The use of new conjugate pairings (Session 17) had no significant effect on either mean RT or the familiarity effect. In fact, there was rather close agreement between the predicted Session 17 familiarity effect and the $150-\mathrm{msec}$ effect actually obtained. This result rules out certain versions of the argument that the induced decrement in the matching RT of the repeated strings was due solely to facilitation of matching processes and that the encoding processes were not altered. For instance, it rules out the hypothesis that the familiarity effect was due to facilitation of a matching process in which the subject tests each letter position for the specific features of the six differing pairs. It also rules out the hypothesis that the familiarity effect was due to facilitation of a matching process in which the subject matches the letter strings as wholes by testing for only the small set of differing features which have been found to occur. However, a more general refutation of the matching explanation awaits future studies, such as inducing a familiarity effect by repeated visual matching and testing for transfer to perceptual tasks which involve report from brief exposure or rapid visual search.

The vertical presentation transfer session (Session 18) was designed to test the notion that familiarity with a letter string allows it to be processed in terms of visual units which are larger than single letters. If such between-letter units are formed during the horizontal presentations of the training sessions, they should be at least partially broken when the strings are presented vertically, and thus the size of the familiarity effect would be expected to be smaller. Unfortunately, the results of Session 18 were ambiguous. The vertical presentation of the letter strings increased RT significantly, but did not cause a significant change in the size of the familiarity effect. However, it is possible that the failure to find statistical significance in the $47-$ msec difference between predicted and obtained familiarity effects is due to the large amount of variability between the four sub- 
jects for Session 18. In fact, the 114-msec familiarity effect found for the vertically presented letter strings is itself only marginally significant, $t(3)=2.81$, $p<.10$, two-tailed.

For the partially familiar letter strings (Session 19), the mean RT was not found to differ significantly from the mean RT of Sessions 13-16. However, the important result of Session 19 is that letter strings with familiar trigrams in familiar positions were matched an average of $103 \mathrm{msec}$ faster than those with familiar trigrams in unfamiliar positions, $F(1,3)=22.10, p<.025 .^{6}$ This result suggests that information about the most likely position of letter sequences is stored during extended perceptual experience and can be used to facilitate the visual matching task.

The marginally significant increase in the familiarity effect for the retention baseline session (Session 20) could indicate that the transfer sessions served as further perceptual training sessions for the repated strings. There were significant familiarity effects for the 1- and 7-week retention sessions $[t(3)=4.14, p<.05 ; t(3)=3.60, p<.05]$, and, furthermore, the sizes of these effects did not differ significantly from the predicted Session 17 familiarity effect. Thus, the effects of stimulus repetition during the training period proved to be relatively longlasting.

An interesting result of the forced-choice recognition task given after Session 16 was that not one subject correctly recognized all six of the repeated letter strings. Several subjects remarked that their recognition performance would have been much better if the letter strings had been presented on the

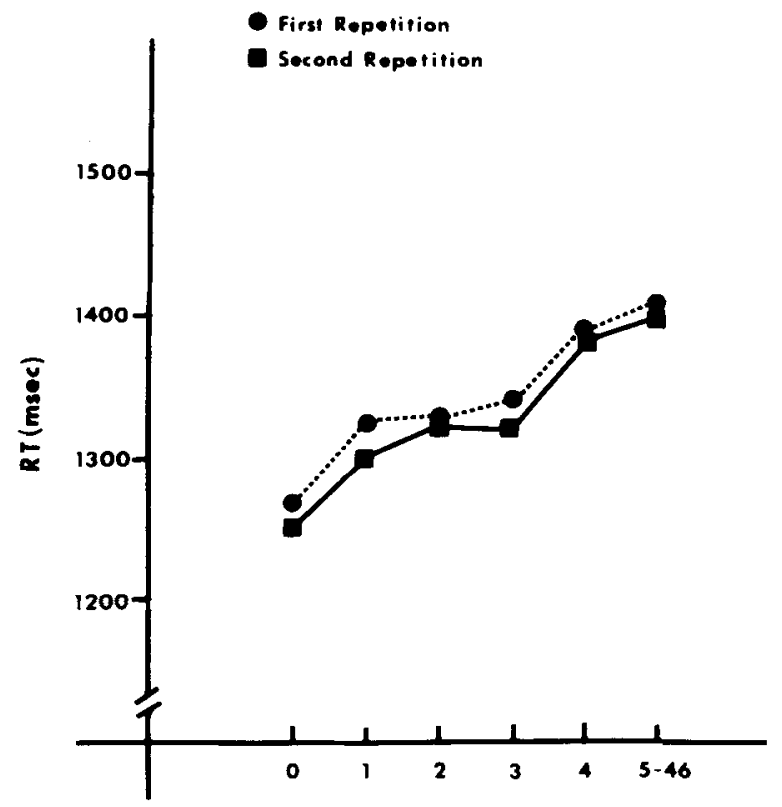

Figure 4. The relationship of matching time to within-block string repetition. display scope rather than typed on a page. Also, there appeared to be a tendency for the novel strings that were incorrectly chosen to have more letters in common with the correct repeated strings than did the average novel string used in the recognition test.

\section{Short-Term Repetition Effects}

Within a single trial block of this study, each nondiffering pair of letter strings was repeated an average of four times (see Note 1). To assess the effect of this within-block repetition, the RTs of nondiffering pairs of letter strings were grouped according to (1) how many previous times the pair had been presented within a trial block and (2) the number of intervening trials, lag, between the pair and its most recent previous occurrence. (All items with lag greater than 4 were pooled.) There was an effect of lag, $F(5,15)=5.79, p<.005$, and a small (14-msec) advantage of second repetitions (i.e., third appearance of a stimulus in a trial block) over first repetitions, $F(1,3)=16.77, p<.05$, but there was no significant interaction of the two effects (see Figure 4).

The fact that the facilitation due to within-block repetition persisted beyond immediate repetitions indicates that, although the effect was short-lived, it was not merely facilitation caused by making the identical response (i.e., pressing a key with the left hand) twice in immediate succession. Interestingly, the effects of within-block repetition were smaller for the repeated strings than for the nonrepeated strings. The effect of lag was smaller for repeated than for nonrepeated stimuli, $F(5,15)=3.80$, $p<.025$ (the difference in RT between lag 0 and lag 5-46 was $64 \mathrm{msec}$ greater for nonrepeated than for repeated stimuli), and the advantage of second over first within-block repetitions was $22 \mathrm{msec}$ smaller for repeated than for nonrepeated strings, $F(1,3)=102.78, p<.005$. Using the logic of the additive factors method (Sternberg, 1969), this interaction between the effects of within-block repetition and the more long-lasting between-session repetition effects suggests that both kinds of repetition act on the same perceptual processes.

\section{Error Rates}

The mean error rate for all subjects over all conditions was $3.23 \%$. An analysis of variance performed on the arcsine transformation of the error rates of each subject in each condition indicated that significantly more errors were made on pairs of differing stimuli than on pairs of identical strings, $F(1,3)=24.17, p<.025$. The mean error rates for repeated and nonrepeated strings were $3.10 \%$ and $3.35 \%$, respectively. Although the difference between them was not significant, the direction of the difference rules out the possibility that the lower 
mean RT for the repeated strings was due to subjects having different speed-accuracy tradeoffs for repeated and nonrepeated strings.

\section{GENERAL DISCUSSION}

With 16 sessions of training, the advantage of repeated strings over nonrepeated strings increased by more than $100 \mathrm{msec}$. Furthermore, this effect did not decrease significantly for more than 7 weeks after the last training session. These results show our procedure to be a laboratory manipulation of familiarity which is effective in inducing relatively long-lasting facilitation of letter strings in a perceptual task.

The fact that sheer repetition of six-letter consonant strings can produce a familiarity effect demonstrates that the perceptual facilitation of familiar strings does not depend solely on their being experienced in a linguistic context (e.g., reading and writing). Also, the induction of a familiarity effect in the present study suggests that a perceptual advantage of familiar strings over unfamiliar strings can exist without differences in their pronounceability (i.e., the size of their acoustic representations). The linear increase in the RT advantage of the repeated letter strings suggests that the familiarity effect develops slowly, but steadily, under conditions of regular perceptual experience. In fact, a previous finding (Well, Pollatsek, \& Schindler, 1975) of a 550-msec difference between words and unpronounceable nonwords for six-letter strings suggests that the increase of the familiarity effect observed over the 16 sessions would have continued if the perceptual training had been maintained.

The present results lead us to several tentative conclusions about the processes used in the visual matching task and how these processes can change with experience. The lack of change of the relative discriminability of the conjugate pairs with experience and the transfer of perceptual training on horizontal strings to vertically presented strings both suggest that perceptual experience with a letter string does not increase the size of the visual units used to process the string. The increase in $R T$ with the increasing serial position of the differing letter pair suggests that the strings were matched by a process which serially compared these letter-size units from left to right and initiated a response when a differing letter pair was found. Furthermore, the increase in the familiarity effect for different responses with increasing position of the difference and the larger familiarity effect for same responses suggests that, with sufficient exposure to a letter string, the processing of the leftmost letters can come to facilitate the processing of the succeeding letters in the string. The finding that familiar trigrams in familiar positions in the string are matched more rapidly than the same trigrams in unfamiliar positions suggests that not only information about what letters follow others, but also information about the position of the letter sequences in the string can be used to facilitate perceptual processing.

In general, the present study has failed to find evidence for qualitative changes in letter-string processing with perceptual experience. It may be that experience does not change the sequence of the visual processes used, but only allows the spquence to occur more rapidly. This idea is especially interesting in light of Piaget's (1969) notion that perceptual learning involves the "sedimentation" of repeated sequences of mental processes. It would seem that methods which manipulate familiarity directly, such as the induction technique used in the present study, are particularly well suited for the further investigation of the nature of the processing changes which occur in perceptual learning.

\section{REFERENCES}

Baron. J. Facilitation of perception by spelling constraints. Canadian Joumat of Psychology. 1974, 28, 37-50.

BARON. I. The word superiority effect. In W. K. Estes (Ed.). Handbosk of learning and cognitive processi's. Potomac. Md: Laurence Erlhaum Associates, in press.

BARON. J.. \& ThuRston, I. An analysis of the word-superiority effect. Cognitive Psychology. 1973, 4, 207-288.

Eichelman. W. H. Familiarity effects in the simultaneous matching task. Journal of Experimental Psycholog.y 1970. 86. $275-282$.

Giвson. E. J. Principles of perceptual leaming and dev'lopment. New York: Appleton-Century-Crotts. 1969.

HENDERSON. L. A word superiority effect without orthographic assistance. Quarterly Joumal of Experimental Psychology. 1974. 26. $301-311$.

KrLeger. L. E. Familiarity effects in visual information processing. Psychological Bulletin. 1975, 82. 949-974.

PAaget. J. The mechanisms of perception. New York: Basic Books. 1969.

REICHER. G. M. Perceprual recognition as a function of the meaningfiulness of the material. Journal of Experimental Psycholog1. 1969, 81. 275-280.

Schindler. R., Well. A.. \& Pollatsek. A. Effects of segmentation and expectancy on matching time for words and nonuords. Jourmal of Experimental Psychology. 1974. 103. $107-111$.

Smith. E. E.. \& Spoerr. K. T. The perception of printed English: A theoretical perspective. In B. Kantowitz (Ed.). Human information processing: Tutorials in performance and cognition. Potomac, Md: Erlbaum Press, 1974

Sternberg. S. Mentory-scanning: Mental processes revealed by reaction-time experiments. American Scientist. 1969. 57. 421-457.

Well, A., Pollatsek, A., \& Schindler, R. Facilitation of both "same" and "different" judgments of letter strings by familiarity of letter sequence. Perception \& Psychophysics. 1975.17 .511 .520 .

\section{NOTES}

1. A "repeated" letter string is one which remained constant over the course of a subject's training and retention sessions, 
while the "nonrepeated" strings were generated anew before each trial block. However, both the repeated and nonrepeated strings appeared several times within a trial block. To avoid confusion, this latter form of repetition will always be referred to as "within-block repetition."

2. The fourth transfer session (Session 21) was an attempt to use the probe-recognition task (Reicher, 1969) to verify the results found with the matching $\mathrm{RT}$ task. Although the accuracy on the repeated strings was greater than that on the nonrepeated strings, this difference was not significant. However, due to the experimenter's error, the stimulus durations were overly long, and it is likely that ceiling effects distorted these results.

3. Since the nonrepeated strings were randomly generated by computer, it was possible, although extremely unlikely, that one of the distractor strings in the forced-choice recognition test was also used as a nonrepeated string during the training sessions.

4. The nonzero intercept of the line in Figure 5 could be due to the four sets of repeated strings happening, by chance, to be more discriminable than the average nonrepeated string. However, it could also have been due to the letter order being more constrained in the repeated strings than in the nonrepeated strings.
Baron (1974) found that such constraints could cause improved performance in a perceptual task even within a single experimental session.

5. Since the familiarity effect tended to increase linearly over the training sessions, the linear projection was used for comparison with the transfer and retention sessions. However, since RTs decreased until around Session 13 and then tended to level off, the RT average of the last four training sessions (rather than the linear projection) was felt to be a more appropriate comparison measure.

6. While the effect of familiar trigram position was larger for letters seen in the first three positions during training (ABCXXX vs. XXXABC) than for those seen in the last three positions during training (XXXDEF vs. DEFXXX), the trigrams which were in familiar positions were matched more rapidly in both cases. However, due to lack of power, only the combined effect was significant.

(Received for publication September 26, 1975; revision accepted February 16, 1976.) 